\title{
ANÁLISIS DE VARIABLES E INDICADORES PARA MEDIR EL E-GOBIERNO PUBLICADOS EN LA LITERATURA CIENTÍFICA
}

\author{
ANALYSIS OF VARIABLES AND INDICATORS \\ FOR MEASURING E-GOVERNMENT PUBLISHED \\ IN SCIENTIFIC LITERATURE
}

Dayanis Esther Pacheco Redondo*

Ernesto Amaru Galvis-Lista"

Mayda Patricia González-Zabala“*

Fecha de recepción: 8 de septiembre de 2016

Fecha de revisión: 6 de abril de 2017

Fecha de aprobación: 5 de mayo de 2017

Cómo citar: D. E. Pacheco Redondo, E. A. Galvis-Lista y M. P. González-Zabala, "Análisis de variables e indicadores para medir el e-gobierno publicados en la literatura científica," Ciencia e Ingeniería Neogranadina, vol. 27,

no. 2, pp. 83-107. DOI: http://dx.doi.org/10.18359/rcin.2226

\section{RESUMEN}

El desarrollo del e-gobierno ha sido promovido como estrategia para mejorar la eficiencia y eficacia de la gestión pública, facilitar la realización de trámites ante las entidades del Estado, ahorrar tiempo y dinero, aumentar la transparencia, acercar al Gobierno a los diferentes actores, así como buscar una mayor participación en la toma de las decisiones. Dada la

\footnotetext{
* Ingeniera de Sistemas. Pasante del Grupo de Investigación y Desarrollo en Tecnología de Información y Organizaciones, de la Universidad del Magdalena, Santa Marta, Colombia.

Correo electrónico: dayanispachecoer@miunimagdalena.edu.co. ORCID: http://orcid.org/0000-0002-5324-299X

** Ingeniero de Sistemas; magíster en Informática y doctor en Ingeniería. Profesor asociado, de la Universidad del Magdalena, Santa Marta, Colombia. Correo electrónico: egalvis@unimagdalena.edu.co. ORCID: http://orcid.org/0000-0002-6012-668X

*** Ingeniera de Sistemas; magíster en Informática y doctora en Ingeniería. Profesora asociada, de la Universidad del Magdalena, Santa Marta, Colombia. Correo electrónico: mpgonzalez@unimagdalena.edu.co. ORCID: http://orcid.org/0000-0002-6847-7919
} 
importancia que el e-gobierno tiene para varios países, incluida Colombia, es importante analizar qué aspectos son considerados, por la comunidad académica y científica, para su medición y seguimiento. En ese sentido, el propósito de este artículo es presentar el resultado del análisis de variables e indicadores propuestos en la literatura científica en el periodo de 2004 a 2014 para medir el gobierno electrónico. Además, se realizó un análisis cientométrico para la literatura publicada en 2015 y 2016. Para identificar los documentos por analizar se realizó una revisión sistemática de literatura. Posteriormente, las variables y los indicadores fueron clasificados y analizados. Los resultados obtenidos evidencian el interés en medir aspectos relacionados con los servicios ofertados en el e-gobierno y el uso de estándares para el desarrollo de los portales, así como en medir aspectos relacionados con la caracterización de usuarios. Los resultados obtenidos pueden constituirse en un punto de partida para la construcción de sistemas de medición del e-gobierno.

Palabras clave: E-gobierno, evaluación, procesos, indicadores, variables.

\section{ABSTRACT}

The development of e-government has been promoted as a strategy to improve: the efficiency and effectiveness of public administration, facilitate the conduct of proceedings, saving time and money, increase transparency, close the government with the different actors, and seek greater participation in decision making. Given the importance of e-government has for several countries, including Colombia, it is important to analyze what aspects are considered, by the academic and scientific community, for measuring and monitoring its development. In that sense, the purpose of this article is to present the analysis of variables and indicators published in the scientific literature in the period of 2004-2014 to measure the e-government. In addition, a scientometric analysis was performed for the literature published in 2015 and 2016. To identify the documents to analyze a systematic literature review was conducted. Subsequently the variables and the indicators were classified and analyzed. The results show the interest in measuring aspects of the services offered on the e-government and the use of standards for the development of portals. In addition, the characterization of users was identified as an important aspect in the measurement process. The results can become a starting point for building measurement systems of e-government.

Keywords: E-government, evaluation, processes, indicators, variables.

\section{INTRODUCCIÓN}

La incorporación de tecnologías de la información y la comunicación (TIC) en diferentes ámbitos de la sociedad, para generar desarrollo social y económico a través del uso de la información y conocimiento, ha configurado un tipo de sociedad que se ha denominado sociedad de la información (SI) [1]. Esta denominación también se asocia a 
los términos sociedad del conocimiento, sociedad digital, sociedad informacional, sociedad en red, economía digital y cibersociedad [2]. Desde un concepto más moderno, la SI "está determinada por una nueva forma de organización económica y social motivada por el desarrollo de las TIC" [3, p. 250]. En este sociedad, se da una expansión y crecimiento del uso de las TIC, evidenciada en la creación de productos y servicios basados en información en todos los ámbitos de la sociedad, por ejemplo, en la salud, la educación, el turismo, entre otros [1].

En este contexto, los Gobiernos de varios países han desarrollado estrategias para la incorporación de las TIC con el fin de obtener los beneficios de la SI, particularmente, con el fin de mejorar la gestión pública. Esta línea de acción se ha denominado gobierno en línea o e-gobierno (e-government). De acuerdo con la revisión de definiciones de e-gobierno propuestas en los contextos académico, gubernamental y empresarial, realizada por [4], [5], se encontró que el e-gobierno busca mejorar la eficacia y eficiencia en el quehacer gubernamental, ofrecer más servicios y posibilitar la realización de trámites ante las entidades del Gobierno, brindar un mejor servicio a los ciudadanos, ahorrar tiempo y dinero, aumentar la transparencia y la divulgación de la información, propender a mejorar los vínculos entre los ciudadanos, empresarios y gobierno, buscar una mayor participación ciudadana en la toma de decisiones y transformar los servicios públicos del Gobierno.

Para impulsar el e-gobierno se debe dar un conjunto de condiciones necesarias para su desarrollo. De acuerdo con [6], en la implementación del e-gobierno es impor- tante tener en cuenta los siguientes aspectos de contexto:

- Normatividad: asociada a los lineamientos, metodologías y normatividad que oriente a las instituciones a desarrollar acciones de e-gobierno.

- Recursos: hace referencia a los recursos que se deben destinar para el desarrollo de tecnología, adecuación de procesos y servicios, entre otros.

- Promoción y divulgación: son los mecanismos para promocionar y divulgar a la sociedad los objetivos y acciones puntuales del e-gobierno.

- Esquema de atención al ciudadano: es la estructura de las entidades gubernamentales para prestar los servicios de gobierno en línea.

- Capacitación: son las orientaciones que la comunidad y las entidades requieren para poder hacer uso de los servicios que se ofrecen.

Asimismo, en la revisión de [4] se identificó que para desarrollar el e-gobierno se deben considera los siguientes aspectos técnicos:

- Infraestructura de TIC: dispositivos de hardware, conectividad, interoperabilidad, implementación de múltiples canales, centros de datos, entre otros.

- Accesibilidad: posibilidad de acceso para todos los usuarios.

- Usabilidad: facilidad de uso.

- Estándares del sitio web: requerimientos funcionales y no funcionales de las entidades del Estado para el diseño de sus sitios web y sus políticas. 
- Satisfacción: la satisfacción del usuario cuando utiliza las aplicaciones y los servicios, y visita sitios web gubernamentales.

- Servicios en línea: servicios en medios electrónicos prestados por las entidades públicas para los ciudadanos, los empresarios y los mismos servidores públicos.

Además de los aspectos normativos, técnicos y de disponibilidad de recursos, un factor esencial para desarrollar el e-gobierno es la participación de los ciudadanos. Es importante pensar sus condiciones particulares en relación con aspectos socioeconómicos y personales, para así enfocar las estrategias de gobierno en línea y lograr su participación [7].

Considerando la relevancia e implicaciones que tiene el e-gobierno, los Gobiernos de varios países se han dado a la tarea de desarrollar este sector de la sociedad de la información. Así mismo, diferentes organismos nacionales e internacionales, gobiernos e investigadores han propuesto sistemas de medición de los avances en la implementación del e-gobierno [8]. Por lo tanto, surge el interés de conocer los aspectos considerados por la comunidad académica y científica para la medición del e-gobierno. En este sentido, el presente artículo muestra los resultados de una revisión sistemática de publicaciones científicas sobre evaluación de e-gobierno, en la cual se identificaron los factores que son evaluados. Para dar cuenta de este trabajo, el artículo se ha estructurado en cuatro secciones. Se inicia con la descripción de la metodología utilizada, luego se presentan los resultados obtenidos y seguidamente las conclusiones; finalmente, se presentan las referencias bibliográficas utilizadas.

\section{METODOLOGÍA}

Para la revisión sistemática de literatura se tomó el método utilizado por [9] para el análisis de las publicaciones realizadas en el periodo de 2004 a 2014. Por otra parte, para las publicaciones realizadas en 2015 y 2016 se realizó un análisis cientiométrico que reporta aspectos generales de la producción científica, como el número de publicaciones, el país donde se desarrollan, entre otras. Para realizar dicho análisis se tomaron como guía de referencias los aspectos definidos en las etapas propuestas por [9], las cuales se presentan a continuación.

\subsection{Planificación}

En esta etapa se desarrolla el protocolo para la revisión sistemática, y se establece así el alcance y los objetivos de la revisión. También, se definieron las preguntas de investigación, los criterios de inclusión/exclusión de documentos, la evaluación de calidad, los procedimientos para la extracción y síntesis de los datos. Las preguntas de investigación que guiaron el proceso de revisión fueron: ¿cuántos artículos científicos se han publicado relacionados con la evaluación del e-gobierno?, ¿para cuáles contextos se han realizado dichas propuestas?, ¿qué variables e indicadores se han propuesto para medir los factores y aspectos relacionados con el e-gobierno?

\subsection{Búsqueda}

La estrategia se centró en realizar la búsqueda de las publicaciones en la base de datos Scopus. El periodo establecido para la bús- 
queda fue de 2004 a 2014; además, se definió la ecuación de búsqueda compuesta por las palabras y sintagmas clave construidos a partir de las definiciones de e-gobierno. Después de 18 iteraciones, la ecuación de búsqueda definitiva se conformó con 153 términos, como se presenta en la Tabla 1. El resultado de aplicar la ecuación de búsqueda en la base de datos seleccionada fue de 13.741 referencias.

Tabla 1. Ecuación de búsqueda

\begin{abstract}
( "assessment" OR "evaluation" OR "review" OR "Measurement" OR "measuring" OR "assess" OR "evaluate" OR "indicators" OR "indicators" OR "gauge" OR "register" OR "gage" OR "telltale" OR "factors" OR "mensuration" OR "methods" OR "procedures" OR "task" OR "activities" OR "practices" OR "scala" OR "integration process" OR "dissemination process" OR "diffusion process" OR "Metrics system" OR "Factor" OR " metrics system tof measurement " OR "metriction" OR "metrics magnitudee" OR " Scale" ) AND ( "e-government" OR "Government online" OR "electronic government" OR "government internet" OR "open government" OR "egov" OR "E-GOV" OR "egovernment" OR "e-governments" OR "E-governance" OR "Public Administration online" OR "E-administration" OR "service oriented government" OR "SERVICE PUBLIC" OR "E-services" OR "G2B" OR "G2C" OR "G2E" OR "G2EI" OR "G2G" OR "IntOGo" OR ( "government" AND "citizens" AND ( internet OR online ) ) OR "E-society" OR "electronic society" OR "E-Citizens" OR "electronic Citizens" OR "e-participation" OR "electronic participation" OR "E-TAXES" OR "electronic tagses" OR "e-audit" OR "electronic unit" OR "e-democracy" OR "electronic democracy " OR "e-democratic " OR "electronic democratic " OR "E-voting " OR "electronic voting " OR "e-Invoice" OR "electronic Invoice" OR "e-business" OR "electronic business" OR "e-justice" OR "electronic justice" OR "e-participation" OR "electronic participation " OR "E-parliament" OR "electronic parliament " OR ( government AND ( "ICT" OR "TIC" OR "Information and communications technology" ) ) ) OR ( ( "Evaluación" OR "crítica" OR "revisión" OR "medición" OR "media" OR "indicatores" OR "mediator de registro " OR " registro" OR " factors" OR " medición " OR " métodos " OR "procedimientos " OR " area " OR " actividades " OR "prácticas " OR " escala " OR "procesos de integración" OR "procesos de difusión" OR "sistema de métricas" OR "Factor" OR "sistema de métricas de medida" OR "sistema métrico" OR "Métrica" OR "magnitude" ) AND ( "e-gobierno" OR "Gobierno en línea" OR "gobierno electrónico" OR "internet Gobierno" OR "gobierno abierto" OR "administración electrónica" OR "E-gobernabilidad " OR "Administración Pública en línea " OR " e-administración " OR "servicio orientada al gobierno " OR " servicio público " OR "e-servicio" OR " servicio electrónica" OR ( "gobierno" AND "ciudadanos "AND ( "internet" OR "línea" ) ) OR "e-sociedad " OR " sociedad electrónica" OR " E-ciudadanos " OR " ciudadanos electrónica" OR " E-participación " OR " participación electrónica" OR " e-impuesto " OR "impuesto electrónica" OR " e-auditoria " OR "auditoria electrónica" OR " e-democracia " OR "democracia electrónica" OR " e-voto" OR " voto electrónica" OR " e-factura " OR " factura electrónica" OR " e-NEGOCIOS " OR " NEGOCIO electrónica" OR " e-Justicia " OR "justicia electrónica" OR " e-parlamento " OR "parlamento electrónica" OR ( "gobierno" AND ( "TIC" OR "TIC" OR "tecnología de la información y comunicaciones ")) ))
\end{abstract}

Fuente: elaboración propia.

\subsection{Selección}

En esta etapa se hizo una selección preliminar a partir de la revisión del título y el resumen del documento, seleccionando aquellos que hacen una mención explícita a la evaluación de e-gobierno o a la propuesta de indicadores de este. El resultado de dicha actividad fue un conjunto de 616 documentos, es decir, el $4,48 \%$ de los identificados. Posteriormente, se realizó una selección detallada de documentos con base en una lectura preliminar del texto completo. La revisión dio como resultado 178 documentos. 


\subsection{Evaluación de calidad}

En esta etapa fue evaluada la calidad de los 178 documentos seleccionados, para lo cual se tuvieron en cuenta cuatro criterios: relevancia del contenido para dar respuesta a la pregunta de investigación definida, descripción detallada del contexto donde se realiza la investigación reportada en el documento, claridad y rigor en el diseño metodológico de la investigación, y rigor científico para el análisis de los datos. El resultado obtenido fue un conjunto de 84 documentos que cumplían con los criterios establecidos. En la Tabla 2 se presenta el resumen cuantitativo de los resultados obtenidos en cada etapa y en la Tabla 3, la relación de documentos que cumplen los criterios de calidad por año.

Tabla 2. Resumen cuantitativo de la búsqueda, selección y evaluación de calidad

\begin{tabular}{|l|l|}
\hline \multicolumn{1}{|c|}{ Etapa } & Total de documentos \\
\hline Búsqueda & 13741 \\
\hline Selección preliminar & 616 \\
\hline Selección detallada & 178 \\
\hline Ecuación de calidad & 84 \\
\hline
\end{tabular}

Fuente: elaboración propia.

Tabla 3. Documentos que cumplen los criterios de calidad por año

\begin{tabular}{|l|l|l|}
\hline Año & No. documentos & \multicolumn{1}{c|}{ Referencias } \\
\hline 2004 & 9 & {$[10]-[18]$} \\
\hline 2005 & 16 & {$[19]-[34]$} \\
\hline 2006 & 7 & {$[35]-[41]$} \\
\hline 2007 & 17 & {$[42]-[58]$} \\
\hline 2008 & 4 & {$[59]-[62]$} \\
\hline 2009 & 3 & {$[63]-[65]$} \\
\hline
\end{tabular}

\begin{tabular}{|l|l|l|}
\hline \multicolumn{1}{|c|}{ Año } & No. documentos & \multicolumn{1}{|c|}{ Referencias } \\
\hline 2010 & 3 & {$[66]-[68]$} \\
\hline 2011 & 1 & {$[69]$} \\
\hline 2012 & 3 & {$[70]-[72]$} \\
\hline 2013 & 12 & {$[73]-[84]$} \\
\hline 2014 & 8 & {$[85]-[92]$} \\
\hline
\end{tabular}

Fuente: elaboración propia.

\subsection{Extracción de datos y síntesis}

En esta etapa final se buscó la extracción de datos para responder las preguntas de investigación definidas. Además, se extrajeron los siguientes metadatos de los registros bibliográficos: título, año de publicación, nombre de la publicación, tipo de documento, autores y país de la institución a la que fue adscrito. A continuación, se presentan los resultados obtenidos.

\section{RESULTADOS}

\subsection{Aspectos generales}

De los 84 documentos seleccionados, se encontró que 29 eran artículos en revistas y 55 artículos en conferencias; es decir, el $34,52 \%$ y el $65,48 \%$, respectivamente. Por otra parte, al indagar cuál era el origen de la propuesta de evaluación, se encontró que la mayor cantidad de documentos proceden de Estados Unidos, con un total de 16 documentos (19\%), seguido de Reino Unido y España, cada uno con el $7 \%$. Países como Taiwán, Brasil, China y Malasia aportan cada uno el $5 \%$ de los documentos, en tanto que Australia, Turquía, Italia y Grecia aportan 
cada uno el $4 \%$. El restante $31 \%$ de los documentos se distribuye en 22 países. En la
Fig. 1 se puede apreciar la distribución de los documentos por país.

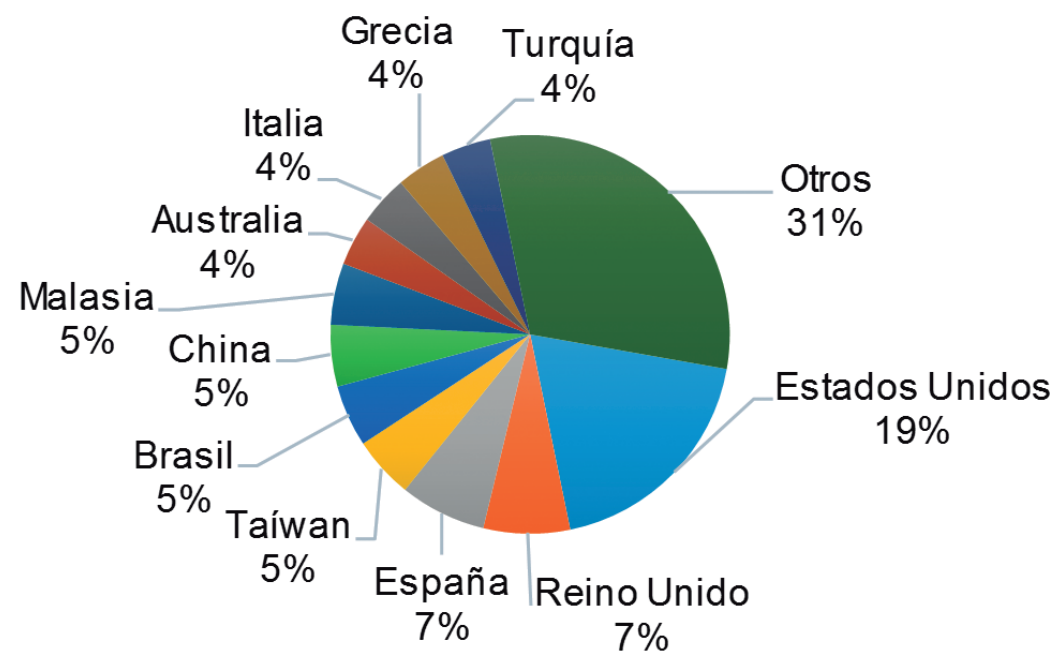

Fig. 1. Distribución de los documentos seleccionados por país de origen Fuente: elaboración propia.

Asimismo, se indagó cuál era el ámbito de aplicación de las propuestas de evaluación de e-gobierno identificadas en los 84 documentos seleccionados. El análisis mostró que el $53,6 \%$ de las propuestas fueron realizadas para un país específico, siendo Estados Unidos el país con más propuestas (11).
Para el contexto de la Unión Europea (UE) y a nivel mundial se identificó el $7,2 \%$ de las propuestas; para Iberoamérica, el 2,3\%, y para Latinoamérica y países de Europa, el 2,4\%. Para el $34,5 \%$ de las propuestas no se identificó el ámbito de aplicación. En la Tabla 4 se aprecia la distribución descrita.

Tabla 4. Ámbito Aplicación

\begin{tabular}{|l|l|l|l|}
\hline \multicolumn{1}{|c|}{ Ámbito } & \multicolumn{1}{c|}{ Descripción } & \multicolumn{1}{c|}{ Total } & \multicolumn{1}{c|}{$\%$} \\
\hline Nacional & Aplicación en un solo país & 45 & 53,6 \\
\hline Unión Europea & Aplicación en los países miembros de la UE & 3 & 3,6 \\
\hline Mundial & Aplicación en países de todo el mundo & 3 & 3,6 \\
\hline Iberoamérica & Aplicación en los países de Iberoamérica & 2 & 2,3 \\
\hline Latinoamérica & Aplicación en Latinoamérica & 1 & 1,2 \\
\hline Europa & Aplicación en los países de Europa & 1 & 1,2 \\
\hline No se especifica & No se especifica & 29 & 34,5 \\
\hline
\end{tabular}

Fuente: elaboración propia. 
Al indagar por las fuentes de información definidas en las propuestas de evaluación, se encontró que el $69,1 \%$ utiliza fuentes primarias, como entrevistas, encuestas, sitios web, entre otras, y el $30,9 \%$ de las propuestas, fuentes secundarias tomando datos de otras investigaciones y fuentes externas. Por otra parte, se realizó la indagación acerca de cuáles fueron los tipos de resultados y se encontró que el $48,8 \%$ se orientaba al pronóstico; el 46,4\%, al diagnóstico, y el 4,8\%, a conocer la ubicación en un escalafón.

\subsection{Identificación y clasificación de las variables e indicadores de evaluación de e-gobierno}

Para la identificación y análisis de variables e indicadores reportados en los 84 documentos se tomó como guía la propuesta metodológica de [93], y para el procesamiento de estas mediciones se usó la herramienta desarrollada por [94]. Con esta actividad se identificaron 764 variables e indicadores.

Posteriormente, se procedió a clasificar las variables e indicadores considerando si evaluaban aspectos del entorno o aspectos técnicos que viabilizan el desarrollo del e-gobierno, así como aspectos de los usuarios. Los resultados mostraron que el $34,4 \%$ $(\mathrm{N}=255)$ mide aspectos relacionados con fases de desarrollo del e-gobierno, por lo cual no se consideraron para el presente estudio, y el $66,6 \%(N=509)$ de las variables e indicadores detectados busca medir los factores en estudio. Específicamente, se encontró que hay $276(54,2 \%)$ variables/indicadores detectados para medir los factores técnicos, $135(26,5 \%)$ variables/indicadores para medir factores de entorno y 98 (19,3\%) variables/indicadores para medir características de los usuarios. De igual manera, se detectó que la distribución entre variables e indicadores es cada una del $50 \%$. En la Fig. 2 y en la Tabla 5, respectivamente, se ilustra y se presenta en detalle la distribución descrita.

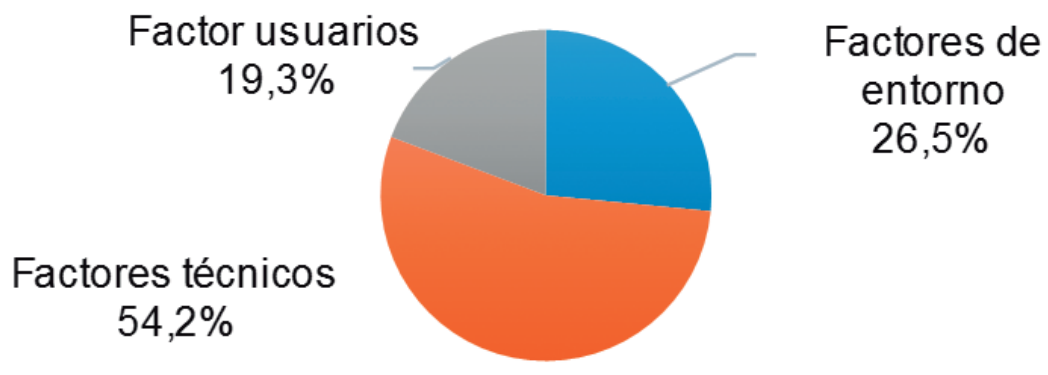

Fig. 2. Distribución de las variables e indicadores identificados en los factores asociados al e-gobierno.

Fuente: elaboración propia. 
Tabla 5. Distribución de indicadores por factores asociados al e-gobierno

\begin{tabular}{|l|l|l|l|}
\hline \multicolumn{1}{|c|}{ Dimensión } & Variable & \multicolumn{1}{c|}{ Indicador } & \multicolumn{1}{c|}{ Total } \\
\hline Entorno & 63 & 72 & 135 \\
\hline Técnicos & 148 & 128 & 276 \\
\hline Usuarios & 43 & 55 & 98 \\
\hline Total & 255 & 254 & 509 \\
\hline
\end{tabular}

Fuente. elaboración propia.

\subsection{Análisis de las variables e indicadores del factor entorno}

Al revisar en detalle los aspectos que son considerados en la medición de los factores de entorno, se encontró que el aspecto relacionado con la asignación de recursos, como los financieros y la asignación de tiempo, tiene el $36,3 \%$ del total de las variables e indicadores, en tanto que el aspecto de la normatividad tiene el $31,1 \%$ de las variables e indicadores. En ete aspecto se evalúan variables asociadas a la existencia de normas jurídicas para la seguridad, privacidad y transparencia de los sitios web públicos, entre otras. El aspecto relacionado con capacitación en e-gobierno tiene el $14,8 \%$ de las variables e indicadores del factor; estos se orientan a medir los materiales, ayudas, cursos, indicaciones de los servicios, entre otras.

El aspecto de promoción y divulgación, que busca medir la eficiencia de la popularidad del sitio, los anuncios, la divulgación, etc., tiene el $14,1 \%$ de las variables e indicadores. Finalmente, se encontró que el $3,7 \%$ de las variables e indicadores miden el esquema de atención al ciudadano, el cual se orienta a la atención del ciudadano, la capacidad de respuesta y los servicios de e-gobierno. En la Tabla 6 se presenta la distribución descrita.

Tabla 6. Distribución de variables e indicadores del factor entorno

\begin{tabular}{|l|l|l|l|}
\hline \multicolumn{1}{|c|}{ Aspecto } & Variables & \multicolumn{1}{c|}{ Indicadores } & Total \\
\hline Recursos & 14 & 35 & 49 \\
\hline Normatividad & 23 & 19 & 42 \\
\hline Capacitación en e-gobierno & 11 & 9 & 20 \\
\hline Promoción y divulgación del e-gobierno & 13 & 6 & 19 \\
\hline Esquema de atención al ciudadano & 2 & 3 & 5 \\
\hline Total & 63 & 72 & 135 \\
\hline
\end{tabular}

Fuente: elaboración propia. 
Por otra parte, se analizó si las variables y los indicadores se orientaban a medir los servicios de e-gobierno desde la oferta y demanda; es decir, los servicios y las condiciones con las cuales se ofrecen, así como la percepción de los usuarios. Cabe destacar que los usuarios pueden ser individuos, hogares o empresas. Este análisis, evidenció que los aspectos de entorno se evalúan mayoritariamente desde la perspectiva de la oferta de servicios con el $94,8 \%$ de las variables e indicadores identificados, mientras que el
$5,2 \%$ se enfoca en medir percepciones de usuarios. Por ejemplo, para el aspecto de normatividad, el indicador utilizado en un enfoque de medición desde la oferta indaga acerca de la existencia de reglamentos que regulen el acceso de servicios, en tanto que el indicador utilizado en un enfoque de medición desde la demanda indaga si el individuo conoce la normatividad referente a e-gobierno. En la Tabla 7 se aprecia la distribución detallada de las variables e indicadores por oferta y demanda.

Tabla 7. Distribución de variables e indicadores del factor entorno por servicio y tipo de usuario

\begin{tabular}{|l|l|l|l|l|l|}
\hline \multirow{2}{*}{ Aspectos } & \multirow{2}{*}{ Servicios } & \multicolumn{3}{c|}{ Usuarios } & \multirow{2}{*}{ Total } \\
\cline { 3 - 6 } & & Empresas & Hogares & Individuos & \\
\hline Recursos & 46 & 0 & 0 & 3 & 49 \\
\hline Normatividad & 41 & 0 & 0 & 1 & 42 \\
\hline Capacitación en e-gobierno & 17 & 0 & 0 & 3 & 20 \\
\hline $\begin{array}{l}\text { Promoción y divulgación del } \\
\text { e-gobierno }\end{array}$ & 19 & 0 & 0 & 0 & 19 \\
\hline $\begin{array}{l}\text { Esquema de atención al } \\
\text { ciudadano }\end{array}$ & 5 & 0 & 0 & 0 & 5 \\
\hline Total & 128 & 0 & 0 & 7 & 135 \\
\hline
\end{tabular}

Fuente: elaboración propia.

\subsection{Análisis de las variables e indicadores del factor técnico}

Para la evaluación del factor técnico se encontró que el aspecto más evaluado es el relacionado con los estándares de sitios web, el cual tiene el $30,15 \%$ de las variables e indicadores, seguido del aspecto de servicios en línea con el 18,1\%. Los aspec- tos de infraestructura y usabilidad tienen el $17,4 \%$ y el $14,5 \%$ de las variables e indicadores del factor, respectivamente. El aspecto de satisfacción tiene el $12,1 \%$ de las variables e indicadores, en tanto que el de accesibilidad tiene el $8 \%$. En la Tabla 8 se presenta la distribución descrita anteriormente. 
Tabla 8. Distribución de variables e indicadores del factor técnico

\begin{tabular}{|l|l|l|l|}
\hline \multicolumn{1}{|c|}{ Aspecto } & Variables & \multicolumn{1}{c|}{ Indicadores } & Total \\
\hline Estándares del sitio web & 42 & 41 & 83 \\
\hline Servicios en línea & 30 & 20 & 50 \\
\hline Infraestructura & 19 & 29 & 48 \\
\hline Usabilidad & 28 & 12 & 40 \\
\hline Satisfacción & 18 & 15 & 33 \\
\hline Accesibilidad & 11 & 11 & 22 \\
\hline Total & 148 & 128 & 276 \\
\hline
\end{tabular}

Fuente. elaboración propia.

Posteriormente, las variables y los indicadores del factor técnico se analizaron teniendo en cuenta su enfoque en la oferta (servicio) o en la demanda (usuarios). Este análisis mostró que el $91,3 \%$ de las variables e indicadores miden los aspectos técnicos de los servicios de e-gobierno ofertados, como el cumplimiento de estándares para el sitio web de la entidad gubernamental, su usabilidad y accesibilidad, su infraestructura, así como la existencia de elementos para medir la satisfacción de los usuarios; mien- tras que el $8,7 \%$ de los indicadores indagan las condiciones de los usuarios, como la tenencia de infraestructura (equipos de cómputo e internet), el grado de satisfacción y su percepción acerca de la accesibilidad y usabilidad. En este análisis, se destaca que se encontraron indicadores que indagan no solo a los individuos, sino también a los hogares y a las empresas, específicamente, en lo relacionado con aspectos de infraestructura. En la Tabla 9 se aprecia la distribución mencionada.

Tabla 9. Distribución de variables e indicadores del factor técnico por servicio y tipo de usuario

\begin{tabular}{|l|l|l|l|l|l|}
\hline \multirow{2}{*}{ Aspectos } & \multirow{2}{*}{$\begin{array}{c}\text { Servicios } \\
\text { ofertados }\end{array}$} & \multicolumn{3}{c|}{ Demanda } \\
\cline { 5 - 7 } & & 82 & 0 & Hogares & Individuos \\
\hline Estándares del sitio web & 49 & 0 & 0 & 1 & 83 \\
\hline Servicios en línea & 38 & 2 & 0 & 1 & 50 \\
\hline Infraestructura & 36 & 0 & 5 & 3 & 48 \\
\hline Usabilidad & & & 3 & 1 & 40 \\
\hline
\end{tabular}




\begin{tabular}{|l|l|l|l|l|l|}
\hline \multirow{2}{*}{ Aspectos } & \multirow{2}{*}{$\begin{array}{c}\text { Servicios } \\
\text { ofertados }\end{array}$} & \multicolumn{2}{c|}{ Demanda } & \multirow{2}{*}{ Total } \\
\cline { 3 - 6 } & & Empresas & Hogares & Individuos & \\
\hline Satisfacción & 28 & 0 & 0 & 5 & 33 \\
\hline Accesibilidad & 19 & 0 & 0 & 3 & 22 \\
\hline Total & 252 & 2 & 8 & 14 & 276 \\
\hline
\end{tabular}

Fuente: elaboración propia.

\subsection{Análisis de las variables e} indicadores del factor usuario

Para este factor se identificaron 43 variables y 55 indicadores, es decir, 98 variables e indicadores que miden aspectos demográficos de los usuarios, como la edad, el nivel de educación, ocupación, entre otras. En este análisis también se identificó que el 56,1\% de las variables e indicadores se centran en caracterizar a los usuarios que acceden a los servicios de e-gobierno, es decir, el análisis se hace desde el acceso al servicio; en tanto que el $60,3 \%$ se orienta a la población en general, buscando medir sus características demográficas, donde específicamente se encontró que el $39,7 \%$ busca medir individuos y el $4,1 \%$, hogares. En la Tabla 10 se presenta la distribución descrita.

Tabla 10. Distribución de variables e indicadores del factor usuario por servicio y tipo de usuario

\begin{tabular}{|l|l|l|l|l|l|}
\hline \multirow{2}{*}{ Aspectos } & \multirow{2}{*}{$\begin{array}{c}\text { Servicios } \\
\text { ofertados }\end{array}$} & \multicolumn{3}{c|}{ Demanda } & \multirow{2}{*}{ Total } \\
\cline { 3 - 6 } & & Empresas & Hogares & Individuos & \\
\hline Características demográficas & 55 & 0 & 4 & 39 & 98 \\
\hline Totales & 55 & 0 & 4 & 39 & 98 \\
\hline
\end{tabular}

Fuente: elaboración propia.

\subsection{Análisis cienciométrico} de publicaciones científicas 2015 y 2016

Con el fin de complementar los análisis realizados de los documentos publicados del 2004 al 2014, se incluyó un análisis cienciométrico para 2015 y 2016 . Este análisis se orienta a identificar el número de publicaciones, la fuente, los autores más destacados y los países donde se realizaron dichos trabajos académicos. Para hacer este análisis, se utilizó la misma ecuación de búsqueda presentada en la Tabla 11 en la base de datos Scopus, considerando únicamente artículos científicos publicados en revistas indexadas 
y conferencias, tal como se realizó el análisis de 2004 a 2014. Como se muestra en la Tabla 11, al realizar la consulta se encontró que el $57,7 \%$ de los documentos correspon- den a artículos en revista indexada y el $42,3 \%$, a artículos de conferencias. También se evidencia el incremento de publicaciones de un año a otro en aproximadamente un $10 \%$.

Tabla 11. Resultado de la ecuación de búsqueda aplicada en Scopus para 2015 y 2016

\begin{tabular}{|l|l|l|l|}
\hline \multicolumn{1}{|c|}{ Año } & \multicolumn{1}{|c|}{ Artículos en revista indexada } & \multicolumn{1}{|c|}{ Artículos en conferencia } & \multicolumn{1}{c|}{ Total } \\
\hline 2015 & 687 & 482 & 1169 \\
\hline 2016 & 727 & 554 & 1281 \\
\hline Total & 1414 & 1036 & 2450 \\
\hline
\end{tabular}

Fuente: Elaboración propia con base en datos de Scopus (20 de marzo de 2017).

Al revisar dichas publicaciones por país cíficamente, Colombia aporta el 0,3\% de se encontró que el 13,8 \% de las publica- las publicaciones. En la Fig. 3 se aprecia la ciones se realizan en Estados Unidos, el distribución del número de publicaciones 9,6 \% en China y el 7,8 \% en Reino Unido. considerando los países que superan las Los países de Latinoamérica aportan el 100 publicaciones y los países latino$6,8 \%$ del total de las publicaciones. Espe- americanos.



Fig. 3. Distribución de publicaciones por país

Fuente: elaboración propia con base en datos de Scopus (20 de marzo de 2017). 
En relación con las instituciones que tienen mayor número de publicaciones reportadas, se encuentra la Delft University of Technology, Brunel University London y Universiti Teknologi Malaysia, cada una de ellas con más de 20 publicaciones. Por otra parte, el top cinco de los investigadores con mayor número de publicaciones en el tema de e-gobierno en el periodo 2015-2016 fueron: Janssen vinculado a Delft University of Technology (Países Bajos), Weerakkody de Brunel University London (Reino Unido), Thiel y Rana de Swansea University (Reino Unido) y Dwivedi del Austrian Institute of Technology (Austria). En la Fig. 4 se puede apreciar la distribución de publicaciones de las instituciones con el mayor número de contribuciones realizadas en el periodo en observación; asimismo, en la Fig. 5 se aprecia la distribución de las publicaciones por los autores con mayor número de contribuciones.
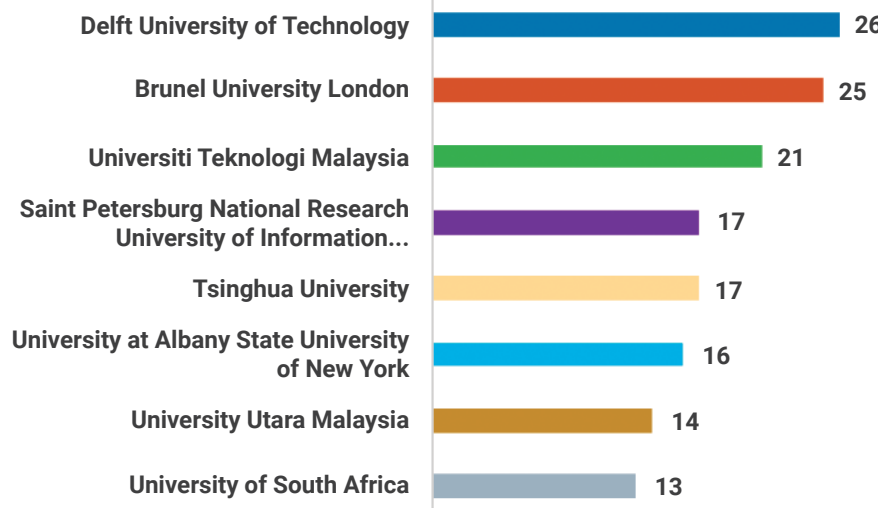

Fig. 4 Distribución de publicaciones de las instituciones con el mayor número de contribuciones. Fuente: elaboración propia con base en datos de Scopus (20 de marzo de 2017).

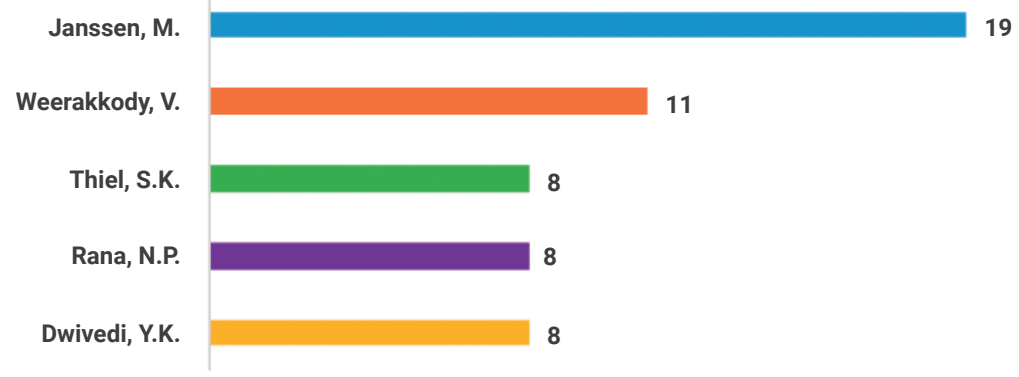

Fig. 5 Distribución de publicaciones de los autores con el mayor número de contribuciones.

Fuente: elaboración propia con base en datos de Scopus (20 de marzo de 2017). 


\section{CONCLUSIONES}

De acuerdo con los resultados obtenidos en el análisis de la literatura académica del periodo de 2004 a 2014, se pudo apreciar que el factor para el cual se ha desarrollado un mayor número de variables e indicadores es el factor asociado con el ciudadano $(19,3 \%)$. Este resultado se puede interpretar como el interés que se tiene por entender el tipo de usuario que emplea los servicios de e-gobierno, con lo cual se pretende con esto caracterizarlos para mejorar los servicios ofertados y ampliar la cobertura.

Asimismo, se detectó que los aspectos relacionados con el factor técnico son los más evaluados, específicamente en lo relacionado con los estándares de sitios web y los servicios en línea. Esto puede indicar el interés que se tiene en conocer cómo va evolucionando la oferta de servicios y el cumplimiento de los mínimos requeridos para los portales web que ofrecen los servicios a los ciudadanos. Estos resultados también pueden evidenciar el interés de ir acercándose al ciudadano y facilitar los trámites. A su vez, se identificó que para la evaluación de la infraestructura se cuenta con un número considerable, aproximadamente el 9,4\% del total de los indicadores, con lo cual se muestran las TIC como un elemento indispensable para el desarrollo de los servicios electrónicos. Además, se identificó que la evaluación de la usabilidad, la accesibilidad y la satisfacción de los usuarios tiene un número importante de variables e indicadores, y con esto se puede inferir el interés que se tiene en evaluar si se facilita el acceso y uso de los servicios ofrecidos.
En relación con la evaluación del factor de entorno, se pudo apreciar que la evaluación de los recursos asignados y la existencia de normatividad que impulse el desarrollo del e-gobierno, con el $17,9 \%$ del total de las variables e indicadores en estudio, son consideradas fundamentales para que se desarrolle el e-gobierno. Además, se resalta el interés de medir aspectos como la capacitación, promoción y divulgación de los servicios de e-gobierno como mecanismo para viabilizar el uso de estos por parte de los ciudadanos y los mismos funcionarios. Por otra parte, se resalta que el número de variables e indicadores para aspectos como el esquema de atención al ciudadano aún es relativamente bajo $(0,9 \%)$ en comparación con otros, esto puede deberse a la dificultad de evaluar dicho aspecto.

Por otro lado, al analizar las variables e indicadores de todos los factores, se encontró que un número significativo de ellos $(94,8 \%$ del factor entorno, el $91,3 \%$ del factor técnico y el $56,1 \%$ del factor usuarios) se enfoca en la medición desde la perspectiva de la oferta del servicio, es decir, busca evaluar los servicios y las condiciones en las que se ponen disponibles los servicios a los ciudadanos; en tanto que es considerablemente bajo el número de las variables e indicadores para medir el uso, las percepciones y las condiciones de acceso de los usuarios. En este sentido, se evidencia que hay pocos elementos para ayudar a determinar la inclusión de los ciudadanos en el e-gobierno, sus condiciones y el uso y percepciones que ellos tienen frente a los servicios ofertados.

Otro aspecto evidente es que al considerar los países que realizan las propuestas 
de medición se identificó que la mayoría se concentran en Estados Unidos. Para el contexto colombiano no se encontró en la literatura científica consultada, ninguna propuesta de medición, aunque cabe destacar que el Gobierno colombiano tiene indicadores propuestos para hacer seguimiento de sus iniciativas [95], como el Índice de Gobierno en Línea [96]. Además, toma como referencia sistemas de medición internacionales, como el World Governance Index, Waseda, Doing Bussines, entre otros [97]. Países como España, Brasil y Reino Unido también tienen producción importante en el tema.

Respecto a lo que se refiere al proceso metodológico utilizado, se evidenció que este permitió la identificación de 84 documentos relacionados con el tema de interés, así como la clasificación y el análisis de las variables e indicadores, con lo que se logró una visión general acerca de los procesos de medición de e-gobierno. Estos resultados permiten tener una base para formular nuevas variables e indicadores que permitan dar cuenta de todos los aspectos relacionados con el e-gobierno y que se pueda comprender con mayor facilidad el funcionamiento de e-gobierno. También, dan un punto de partida para nuevos trabajos investigativos relacionados con el e-gobierno, con el fin de que se pueda comprender con mayor facilidad el funcionamiento y brindar elementos que permitan su desarrollo. Cabe destacar que entre las limitantes de la investigación se encuentra el acceso limitado que se tiene a algunos documentos.

Por último, el análisis cienciométrico de la literatura publicada en 2015 y 2016 permitió evidenciar que el tema sigue vigente, dado que el número de publicaciones ha venido en aumento. Asimismo, se detectó que el liderazgo de algunos países como Estados Unidos y Reino Unido se mantiene. Esto se puede apreciar no solo en el número de artículos que reportan, sino también en las instituciones y los autores que son referentes por sus contribuciones al tema.

\section{Referencias}

[1] J. M. Sánchez-Torres, M. P. González-Zabala y M. P. S. Muñoz, "La Sociedad de la Información: Génesis, Iniciativas, Concepto y su Relación con Las TIC," Revista UIS Ingenierías, vol. 11, no. 1, pp.113-128, 2013.

[2] R. Ruiz de Querol y J. Buira. La sociedad de la información. Barcelona, España: Editorial UOC, 2007.

[3] P. Valenti, "La Sociedad de la Información en América Latina y el Caribe: TICs y un Nuevo Marco Institucional," Revista Iberoamericana de Ciencia, Tecnología, Sociedad e Innovación, vol. 2, 2002. [En línea]. Disponible en: http://www.oei.es/historico/revistactsi/numero2/valenti.htm

[4] D. E. Pacheco y M. P. González-Zabala, "Análisis de definiciones de e-gobierno y sus aspectos asociados," Tesis de pregrado, Facultad de Ingeniería, Ingeniería de Sistemas, Universidad del Magdalena. Santa Marta, Colombia, 2015.

[5] D. E. Pacheco, "Revisión sistemática de la literatura científica sobre los procesos de medición de e-gobierno," Te- 
sis de pregrado, Facultad de Ingeniería, Universidad del Magdalena. Santa Marta, Colombia, 2015.

[6] J. M. Sánchez-Torres, "Propuesta metodológica para evaluar políticas públicas de promoción del e-government como campo de aplicación de la sociedad de la información. El caso colombiano," Tesis de doctorado, Doctorado en Economía y Gestión de la Innovación y Política Tecnológica, Universidad Autónoma de Madrid, Madrid, España, 2006.

[7] Colombia, Ministerio de Tecnologías de la Información y las Comunicaciones, "Guía para la caracterización de usuarios de las entidades públicas," 2011. [En línea]. Disponible en: http://estrategia.gobiernoenlinea.gov. co/623/articles-8536_recurso_1.pdf.

[8] M. P. González-Zabala y J. M. Sánchez-Torres, "Análisis de variables e indicadores empleados para medir la sociedad de la información," Revista Ingeniaré, vol. 20, no. 3, pp. 433-446, 2012. https://doi.org/10.4067/s071833052012000300017

[9] E. Galvis-Lista y J. Sánchez-Torres, "Revisión sistemática de literatura sobre procesos de gestión de conocimiento," Revista Gerencia Tecnológica Informática, vol. 13, no. 37, pp. 45-67, 2014.

[10] J. C. Steyaert, "Measuring the performance of electronic government services," Information and Management, vol. 41, no. 3, pp. 369-375., 2014 https://doi.org/10.1016/S03787206(03)00025-9
[11] D. Janssen, S. Rotthier y K. Snijkers, "If you measure it they will score: An assessment of international eGovernment benchmarking," Information Polity, vol. 9, no. 3-4, pp. 121-130, 2004.

[12] S. Kim y H. Lee, "Organizational factors affecting knowledge sharing capabilities in E-government: An empirical study," en IFIP Int. Working Conf. on Knowledge Management in Electronic Government, pp. 281-293, 2004. https://doi. org/10.1007/978-3-540-24683-1_28

[13] R. M. Peters, M. Janssen y T. M. Van Engers, "Measuring e-government impact: Existing practices and shortcomings," en 6a Int. Conf. on Elec. Commerce, Copenhagen, Dinamarca, 2004, pp. 480-489. https://doi. org/10.1145/1052220.1052281

[14] J. Kaaya, "The emergence of e-government services in East Africa: Tracking adoption patterns and associated factors," en 6a Int. Conf. on Elec. Commerce, Copenhagen, Dinamarca, 2004. pp. 438-445. https://doi. org/10.1145/1052220.1052276

[15] P. Shackleton, J. Fisher y L. Dawson, "Evolution of local government e-services: The applicability of e-business maturity models," en 37th Annu. Hawaii Int. Conf. on System Sciences, Hawaii, Estados Unidos, 2004, pp. 1913-1922. http://dx.doi.org/10.1109/ hicss.2004.1265308

[16] H. J. Kim y S. Bretschneider, "Local government information technology capacity: An exploratory theory," en 37th Annu. Hawaii Int. Conf. on System 
Sciences, Hawaii, Estados unidos, 2004 pp. 1933-1942. http://dx.doi. org/10.1109/hicss.2004.1265310

[17] J.-R. Fu, W.-P. Chao y C.-K. Farn, "Determinants of taxpayers' adoption of electronic filing methods in Taiwan: An exploratory study," Journal of Government Information, vol. 30, no. 5-6, pp. 658683, 2004. https://doi.org/10.1016/j. jgi.2004.11.002

[18] L. A. Joia, "Developing Government-to-Government enterprises in Brazil: A heuristic model drawn from multiple case studies," International Journal of Information Management, vol. 24, no. 2, pp. 147-166, 2004. https://doi.org/10.1016/j.ijinfomgt.2003.12.013

[19] H. Abdelghaffar, W.-E. Bakry y P. Duquenoy, "E-government: A new vision for success," en European and Mediterranean Conf. on Inf. Systems, Cairo, Egypt, 2005, pp. 1-8.

[20] P. Rossel y M. Finger, "Towards a multi-measurement platform of e-Government projects and services," en European Conf. on e-Government - ECEG, 2005, Antwerp, Belgium, pp. 349-358.

[21] Z. J. Kovačić, "The impact of national culture on worldwide eGovernment readiness," Informing Science, vol. 8, pp. 143-158, 2005.

[22] D. Kettani, B. Moulin y A. Elmahdi, "Towards a formal framework of impact assessment of e-Government systems on governance," WSEAS Transactions on Computers, vol. 4, no. 12, pp. 1858-1866, 2005.
[23] C. Caba Pérez, A. M. López Hernández y M. P. Rodríguez Bolívar, "Citizens' access to on-line governmental financial information: Practices in the European Union countries," Government Information Quarterly, vol. 22, no. 2, pp. 258276, 2005. https://doi.org/10.1016/j. giq.2005.02.002

[24] C. Seel, O. Thomas, B. Kaffai y T. Matheis, "Evaluating e-government: A process-oriented approach," en IFIP Conf. 2005, Poznan, Polonia, 2005, pp. 111-124. https://doi.org/10.1007/0387-29773-1_8

[25] M. Qi y B. Wei, "Design and analysis of administrative approval act based electronic government evaluation system," en 7th Int. Conf. on Elec. Commerce - ICEC '05, Xi'an, China, 2005, pp. 553-557. http://dx.doi. org/10.1145/1089551.1089651

[26] B. Li, "On the barriers to the development of e-government in China," en 7th Int. Conf. on Elec. Commerce - ICEC '05, Xi'an, China, 2005, pp. 549-552. http:// dx.doi.org/10.1145/1089551.1089650

[27] T. Zwahr, M. Finger y P. Mueller, "More than digitisation - The transformative potential of e-governance: An exploratory case study," en 38th Annu. Hawaii Int. Conf. on System Sciences, Hawaii, Estados Unidos, 2005, pp. 127. http:// dx.doi.org/10.1109/hicss.2005.443

[28] M. Janssen y A. Cresswell, "Enterprise architecture integration in E-government," en 38th Annu. Hawaii Int. Conf. on System Sciences, Hawaii, Estados 
Unidos, 2005, pp. 118. https://doi. org/10.1109/HICSS.2005.243

[29] L. Torres, V. Pina y S. Royo, "E-government and the transformation of public administrations in EU countries: Beyond NPM or just a second wave of reforms?," Online Information Review, vol. 29 , no. 5, pp. 531-553, 2005. https://doi. org/10.1108/14684520510628918

[30] L. Torres, V. Pina y B. Acerete, "E-government developments on delivering public services among EU cities," Government Information Quarterly, vol. 22, no. 2, pp. 217-238, 2005. https://doi. org/10.1016/j.giq.2005.02.004

[31] M. Paris, "Local e-government and devolution: Electronic service delivery in Northern Ireland," Local Government Studies, vol. 31, no. 3, pp. 307-319, 2005. https://doi. org/10.1080/03003930500095137

[32] L. L. Tung, y O. Rieck, "Adoption of electronic government services among business organizations in Singapore," Journal of Strategic Information Systems, vol. 14, no. 4, pp. 417-440, 2005. https:// doi.org/10.1016/j.jsis.2005.06.001

[33] P. S. Herrnson, B. B. Bederson, B. Lee, P. L. Francia et al., "Early appraisals of electronic voting," Social Science Computer Review, vol. 23, no. 3, pp. 274-292, 2005. https://doi. org/10.1177/0894439305275850

[34] J. C. Thomas y G. Streib, "E-democracy, e-commerce, and e-research: Examining the electronic ties between citizens and governments," Administration and Society, vol. 37, no. 3 , pp. $259-280,2005$. https://doi. org/10.1177/0095399704273212.

[35] T. Carratta, L. Dadayan y E. Ferro, "ROI analysis in e-Government assessment trials: The case of sistema piemonte," Lecture Notes in Computer Science, pp. 329-340, 2006. https://doi. org/10.1007/11823100_29

[36] V. Petricek, T. Escher, I. J. Cox y H. Margetts, "The web structure of e-government - Developing a methodology for quantitative evaluation," en 15th Int. Conf. on World Wide Web, New York, Estados Unidos, 2006, pp. 669-678. https://doi. org/10.1145/1135777.1135875

[37] N. Mohamed, H. Hussin y R. Hussein, "Enabling change factors and IT success in the Malaysian e-government implementation," en PACIS 2006 - 10th Pacific Asia Conf. on Inf. Systems: ICT and Innovation Economy, Taiwan, China, 2006, pp. 1107-1125.

[38] P. T. Jaeger, "Assessing Section 508 compliance on federal e-government Web sites: A multi-method, user-centered evaluation of accessibility for persons with disabilities," Government Information Quarterly, vol. 23, no. 2, pp. 169-190, 2006. https://doi. org/10.1016/j.giq.2006.03.002

[39] N. Braun y D. Brändli, "Swiss e-voting pilot projects: Evaluation, situation analysis and how to proceed," en Elec. Voting 2006 - 2nd Int. Workshop, Castle Hofen, Bregenz, Austria, 2006, pp. 27-36.

[40] P. V. Vora, R. Simha y J. Stanton, "A performance ratings framework 
for the evaluation of electronic voting systems," en The 2006 Nat. Conf. on Digital Government Research - dg.o '06, San Diego, California, 2006, pp. 460-461. http://dx.doi. org/10.1145/1146598.1146749

[41] L. F. Luna-Reyes, J. R. Gil-Garcia y C. B. Cruz, "Collaborative digital government in Mexico: Some lessons from federal web-based inter- organizational information integration initiatives," Government Information Quarterly, vol. 24, no. 4, pp. 808-826, 2007. https:// doi.org/10.1016/j.giq.2007.04.003.

[42] J. Cheng, S. Cheng y M. Yang, "Evaluating the e-government based on BSC," IFIP International Federation for Information Processing, vol. 252, no. 2, pp. 397-403, 2007. https://doi. org/10.1007/978-0-387-75494-9_48

[43] M. Grimsley y A. Meehan, "E-Government information systems: Evaluation-led design for public value and client trust," European Journal of Information Systems, vol. 16, no. 2, pp. 134148, 2007. https://doi.org/10.1057/ palgrave.ejis. 3000674

[44] W. Castelnovo y M. Simonetta, "A public value evaluation of e-Government policies," en ECIME 2007: European Conf. on Inf. Management and Evaluation, Reino Unido, 2007, pp. 63-70.

[45] I. Taoufik, H. Kabaili y D. Kettani, "Designing an E-government portal accessible to illiterate citizens," en 1st Int. Conf. on Theory and Practice of Elec. Governance - ICEGOV '07, Macao SAR,
China, 2007, pp. 327-336. https://doi. org/10.1145/1328057.1328125

[46] A. A. Golubeva, "Evaluation of regional government portals on the basis of public value concept: Case study from Russian federation," en 1st Int. Conf. on Theory and Practice of Elec. Governance - ICEGOV '07, Macao SAR, China, 2007, pp. 394-397. https://doi. org/10.1145/1328057.1328139

[47] B. P. Shah y S. Shakya, "Evaluating the web accessibility of websites of the central government of Nepal," en 1st Int. Conf. on Theory and Practice of Elec. Governance - ICEGOV '07, Macao SAR, China, 2007, pp. 447-448. https:// doi.org/10.1145/1328057.1328154

[48] N. Mitsamarn, W. Gestubtim y S. Junnatas, "Web accessibility: A government's effort to promote e-accessibility in Thailand," en The 1st Int. Convention on Rehabilitation Engineering \& Assistive Technology in Conjunction with 1st Tan Tock Seng Hospital Neurorehabilitation Meeting - i-CREATe '07, Singapore, 2007, pp. 23-27. https://doi. org/10.1145/1328491.1328498

[49] A. Henriksson, Y. Yi, B. Frost y M. Middleton, "Evaluation instrument for e-government websites," Electronic Government, vol. 4, no. 2, pp. 204226, 2007, https://doi.org/10.1504/ EG.2007.013984

[50] P. Dandjinou, "E-Governance strategies in Cape Verde and South Africa: From service delivery to e-participation," en 1st Int. Conf. on Theory and Practice of Elect. Governance - ICEGOV '07, Macao 
SAR, China, 2007, pp. 435-436. http:// dx.doi.org/10.1145/1328057.1328148

[51] T. M. Waema y W. Mitullah, "E-governance and governance: A case study of the assessment of the effects of integrated financial management system on good governance in two municipal councils in Kenya," en 1st Int. Conf. on Theory and Practice of Elec. Governance - ICEGOV '07, Macao SAR, China, 2007, pp. 263-268. https://doi. org/10.1145/1328057.1328113

[52] F. Corradini, F. Marcantoni, A. Polzonetti y B. Re, "A formal model for quality of service measurement in e-Government," en 29th Int. Conf. on Information Technology Interfaces ITI, Cavtat, Croatia, 2007, pp. 747-752. http://dx.doi. org/10.1109/iti.2007.4283865

[53] N. Zhang, X. Guo y G. Chen, "Diffusion and evaluation of e-government systems: A field study in China," en PACIS 2007. 11th Pacific Asia Conf. on Information Systems, Auckland, Nueva Zelanda, 2007 pp. 271- 283.

[54] Y.-H. Hung, Y.-S. Wang y S.-C. T. Chou, "User acceptance of E-Government services," en PACIS 2007. 11th Pacific Asia Conf. on Inf. Systems, Auckland, Nueva Zelanda, 2007, pp. 1-9.

[55] C.C. Yu, "A value-based strategic management process for e-government strategy planning and performance control," en 1st Int. Conf. on Theory and Practice of Elec. Governance - ICEGOV '07, Macao SAR, China, 2007, pp. 169-178. http://dx.doi. org/10.1145/1328057.1328093
[56] S. Alshawi, A. Alahmary y H. Alalwany, "E-government evaluation factors: Citizen's perspective," en Proceedings of the European and Mediterranean Conf. on Inf. Systems, EMCIS 2007, Valencia, España, 2007, pp. 28-1-28-12.

[57] J. Shiang, N. Hsiao y J. Lo, "Democratization of government websites: Indicators and comparing perceptions of citizens and public officials in Taiwan," en The European Conf.e on e-Government, ECEG- 2007, Reino Unido, 2007, pp. 491-499.

[58] Z. Baida, J. Liuy Y.H. Tan, "Towards a methodology for designing e-Government control procedures," Lecture Notes in Computer Science, vol. 4656, 2007, pp. 56-67. http://dx.doi.org/10.1007/978-3540-74444-3_6

[59] M. Shareef, A. Ojo y T. Janowski, "A readiness assessment framework for e-government planning-design and application," en ICEGOV '08 Proceedings of the 2nd Int. Conf. on Theory and Practice of Elec. Governance, Cairo, Egipto, 2008, pp. 403-410. https:// doi.org/10.1145/1509096.1509180

[60] E. Panopoulou, E. Tambouris y K. Tarabanis, "A framework for evaluating web sites of public authorities," Aslib Proceedings: New Information Perspectives, vol. 60, no. 5, pp. 517-546, 2008. https:// doi.org/10.1108/00012530810908229

[61] A. A. Economidesy V.Terzis, "Evaluating tax sites: An evaluation framework and its application," Electronic Government, vol. 5, no. 3, pp. 321-344, 2008. https:// doi.org/10.1504/EG.2008.018878 
[62] S. Hong, P. Katerattanakul y S. J. Joo, "Evaluating government website accessibility: A comparative study," Int. Journal of Inf. Technology and Decision Making, vol. 7, no. 3, pp. 491515, 2008. https://doi.org/10.1142/ S0219622008003058

[63] M. Sorrentino y K. Passerini, "Evaluating the implementation process: An exploratory e-government case study," en 15th Americas Conf. on Inf. Systems, AMCIS - 2009, 2009, pp. 4548-4554.

[64] I. Akdoğan, "Evaluating and improving e-participation in Istanbul," en Proceedings of the 3rd Int. Conf. on Theory and Practice of Elec. Governance - ICEGOV '09, Bogota, Colombia, 2009, pp. 103-108. https://doi. org/10.1145/1693042.1693064

[65] S. Al-Shafi, V. Weerakkody y M. Janssen, "Investigating the adoption of e-government services in Qatar using the UTAUT model," en 15th Americas Conf. on Inf. Systems 2009 - AMCIS 2009, San Francisco, Estados Unidos, 2009, pp. 15-24.

[66] P. Li y G. Viscusi, "Quality evaluation for strategic alignment engineering: An eGovernment application," en $\mathrm{CA} i$ SE 2010 Workshop BUSITAL'10, Hammamet, Túnez, 2010 pp. 1-15.

[67] M. N. Norshita, B. Z. Halimah y T. S. Tengku Mohdammad, "Public user assessment of Malaysia's e-Government applications," International Journal of Social, Behavioral, Educational, Economic, Business and Industrial Engineering, vol. 4, no. 7, pp. 1766-1770, 2010.
[68] E. Loukis, A. Xenakis, R. Peters y Y. Charalabidis, "Using gis tools to support e-participation - A systematic evaluation," Lecture Notes in Computer Science, vol. 6229, pp. 19-210, 2010. https://doi.org/10.1007/978-3-64215158-3_17

[69] G. B. Viana y M. B. F. de Toledo, "Electronic government in Brazil: Evaluating Brazil initiative," en ICEIS 2011 Proceedings of the 13th Int. Conf. on Enterprise Information Systems, Beijing, China, 4 SAIC, 2011, pp. 388-402.

[70] G. P. Adhikari, "Evaluation of E-governance projects of Nepal," en 6th Int. Conf. on Theory and Practice of Elec. Governance-ICEGOV'12. Albany, NY, Estados Unidos, 2012, pp. 472-473. https:// doi.org/10.1145/2463728.2463824

[71] R. Sandoval-Almazan, J. R. Gil-Garcia y N. K. S. Leyva, "E-government portals in central America: A preliminary evaluation and ranking (20112012)," en 6th Int. Conf. on Theory and Practice of Elec. Governance ICEGOV '12. Albany, NY, Estados Unidos, 2012, pp. 426-429. https://doi. org/10.1145/2463728.2463810

[72] M. Alruwaie, R. El-Haddadeh y V. Weerakkody, "A framework for evaluating citizens' expectations and satisfaction toward continued intention to use e-government services," Lecture Notes in Computer Science, vol. 7443, pp. 273-286, 2012. https://doi. org/10.1007/978-3-642-33489-4_23

[73] Y. Lin y S. Fong, "Performance evaluation management model to accelerate 
the development of e-government in China," en 7th International Conference on Theory and Practice of Electronic Governance - ICEGOV '13, Seul, República de Corea, 2013, pp. 1-4. https:// doi.org/10.1145/2591888.2591889

[74] M. M. Yusof y A. Y. A. Yusuff, “Evaluating E-government system effectiveness using an integrated socio-technical and fit approach," Information Technology Journal, vol. 12, no. 5, pp. 894906, 2013. https://doi.org/10.3923/ itj.2013.894.906

[75] D. Stanimirovic y M. Vintar, "A critical insight into the evaluation of e-government policies: Reflections on the concept of public interest," Int. Journal on Advances in Life Sciences, vol. 5, no. 1-2, pp. 52-65.

[76] J. Hughes, P. Ahluwalia y V. Midha, "A heuristic evaluation instrument for e-government online software," Electronic Government, vol. 10, no. 1, pp. 1-18, 2013. https://doi.org/10.1504/ EG.2013.051273

[77] M. Goodwin, "Towards automatic assessment of government web sites," en 3rd Int. Conf. on Web Intelligence, Mining and Semantics - WIMS '13, Madrid, España, 2013. https://doi. org/10.1145/2479787.2479805

[78] A. Bikfalvi, J. L. de La Rosa y T. N. Keefe, "E-Government service evaluation: A multiple-item scale for assessing information quality," en Lecture Notes in Informatics (LNI), Proceedings - Series of the Gesellschaft fur Informatik (GI), 2013, pp. 54-61.
[79] R. Sandoval-Almazan, N. K. Saucedo Leyva y J. R. Gil-Garcia, "Maturity and evolution of e-government portals in central America: A three-year assessment 2011-2013," en 7th Int. Conf. on Theory and Practice of Elec. Governance - ICEGOV '13, Seul, República de Corea, 2013, pp. 182-185. https://doi. org/10.1145/2591888.2591918

[80] A. Al-Faries, H. S. Al-Khalifa, M. S. Al-Razgan y M. Al-Duwais, "Evaluating the accessibility and usability of top Saudi e-government services," en 7th Int. Conf. on Theory and Practice of Elec. Governance - ICEGOV '13, Seul, República de Corea, 2013, pp. 60-63. https:// doi.org/10.1145/2591888.2591898

[81] G. Iskender y S. Özkan, "E-government transformation success: An assessment methodology and the preliminary results," Transforming Government: People, Process and Policy, vol. 7, no. 3, pp. 364-392, 2013. https://doi. org/10.1108/TG-09-2012-0008

[82] A. Tsohou, H. Lee, Z. Irani, V. Weerakkody et. al., "Proposing a reference process model for the citizen-centric evaluation of e-government services," Transforming Government: People, Process and Policy, vol. 7, no. 2, pp. 240-255, 2013. https:// doi.org/10.1108/17506161311325387

[83] C. Pérez-Espés, J. M. M. Jiménez y M. A. Wimmer, "Evaluating the efficacy of e-participation experiences," en Lecture Notes in Informatics (LNI), Alemania, vol. P-221, 2013, pp. 250-257.

[84] S. Ereminaite, "Assessment aspects of electronic public procurement maturity," 
Public Policy and Administration, vol. 12, no. 4, pp. 567-580, 2013.

[85] A. S. Corrêa, P. L. P. Corrêa y F. S. C. da Silva, "Transparency portals versus open government data. An assessment of openness in Brazilian municipalities," en 15th Annu. Int. Conf. on Digital Government Research, Aguascalientes, Mexico, 2014, pp. 178-185. https://doi. org/10.1145/2612733.2612760

[86] V. Venkatesh, H. Hoehle y R. Aljafari, "A usability evaluation of the Obamacare website," Government Information Quarterly, vol. 31, no. 4, pp. 669680, 2014. https://doi.org/10.1016/j. giq.2014.07.003

[87] N. Karkin y M. Janssen, “Evaluating websites from a public value perspective: A review of Turkish local government websites," International Journal of Information Management, vol. 34, no. 3, pp. 351-368, 2014. https://doi. org/10.1016/j.ijinfomgt.2013.11.004

[88] R. Arendsen, O. Peters, M. ter Hedde y J. van Dijk, “Does e-government reduce the administrative burden of businesses? An assessment of business-to-government systems usage in the Netherlands," Government Information Quarterly, vol. 31, no. 1, pp. 160169, 2014. https://doi.org/10.1016/j. giq.2013.09.002

[89] N. P. Rana, S. Ghobadi, Y. K. Dwivedi, N. C. Piercy y M. D. Williams, "Assessing adoption of online PAN card registration system (OPCRS): An Indian e-government system perspective," en 20th Americas Conf. on Information Sys- tems, AMCIS 2014, Savannah, Georgia, Estados Unidos, 2014. pp. 1-10.

[90] J. M. A. P. Cestari, E. R. Loures, E. A. P. Santos y M. Lezoche, "A Research Strategy for Public Administration Interoperability Assessment," en IIE Annu. Conf. Proceedings, Montreal, Canada, 2014, pp. 3134-3143.

[91] M. K. Faaeq, K. Alqasa y E. M. Al-Matari, "Technology adoption and innovation of E-Government in Republic of Iraq," Asian Social Science, vol. 11, no. 3, pp. 135-145, 2015. https://doi. org/10.5539/ass.v11n3p135

[92] F. J. Alcaraz-Quiles, A. Navarro-Galera y D. Ortiz-Rodríguez, "Factors influencing the transparency of sustainability information in regional governments: An empirical study," Journal of Cleaner Production, vol. 82, pp. 179-191, 2014. https://doi.org/10.1016/j.jclepro.2014.06.086

[93] M. P. González-Zabala y J. M. Sánchez-Torres, "Propuesta de una metodología para el diseño de sistemas de indicadores para la evaluación de la Sociedad de la Información en Colombia," en IX Congr. Iberoamericano de Indicadores de Ciencia y Tecnología, Bogotá, Colombia, 9-11 oct. 2013 pp. 1-9.

[94] M. P. González-Zabala y E. A. Galvis-Lista, "Diseño de un sistema de información para el análisis de indicadores de medición de la sociedad de la información," Entre Ciencia e Ingeniería, vol. 0, no. 16, pp. 65-71, 2014.

[95] M. P. González-Zabala, J. M. Sánchez-Torres y J. A. Holbrook, "Análisis 
de los indicadores para medir las iniciativas de Sociedad de la Información propuestas por el gobierno colombiano," Universitas Humanística, vol. 76, no. 76, pp. 277-298, 2013.

[96] Colombia, Ministerio de Tecnologías de la Información y Comunicación, "Índice de gobierno en línea," 2016. [En línea]. Disponible en: http://estrategia. gobiernoenlinea.gov.co/623/w3-propertyvalue-7651.html

[97] Colombia, Ministerio de Tecnologías de la Información y Comunicación, "Índices internacionales de medición de la estrategia de gobierno en línea," 2016. [En línea]. Disponible en: http://estrategia.gobiernoenlinea.gov. co/623/w3-propertyvalue-7653.html. 